Tatsächlich ist seine vormalige Abrechnungsweise jedoch teilweise unrichtig, nämlich im Hinblick auf die Besuchsgebühr.

Die vom Bf. vorgenommene analoge Heranziehung der Nr. 50 des Gebührenverzeichnisses der GOÄ ist unzulässig. Denn nach $\$ 6$ Abs. 2 GOÄ dürfen Analogien nur gebildet werden, wenn selbständige ärztliche Leistungen nicht in das Gebührenverzeichnis aufgenommen sind. Für die Leichenschau stellt das Gebührenverzeichnis der GOÄ indes die Nr. 100 zur Verfügung (,Untersuchung eines Toten einschließlich Feststellung des Todes und Ausstellung des Leichenschauscheines“). Seitens der Zivilgerichtsbarkeit ist denn auch wiederholt - wenn auch ,nur" auf amtsgerichtlicher Ebene - entschieden worden, dass für eine Leichenschau keine Besuchsgebühr nach Nr. 50 des Gebührenverzeichnisses der GOÄ abgerechnet werden dürfe, und zwar weder direkt noch analog (vgl. dazu und zum Folgenden das Urt. des AG Herne-Wanne v. 8.9.1998 - 2 C 380/98und das Urteil des AG Oberhausen vom 19.3.1998 - 37 C 79/98 -. Der Gebührentatbestand der Nr. 50 des Gebührenverzeichnisses der GOÄ (,Besuch, einschließlich Beratung und symptombezogene Untersuchung") erfasst ersichtlich nur eine Leistung am lebenden Patienten. Eine Abrechnung der Besuchsgebühr neben der Leichenschau ist daher nur dann gerechtfertigt, wenn der später Verstorbene zur Zeit der Beauftragung des Arztes noch lebte oder wenn die Angehörigen in Anbetracht des Todesfalls selbst gesundheitlich angegriffen sind und insofern den $\mathrm{R}$ at des Arztes einholen. Jedenfalls mittlerweile gehen auch sowohl die Bundesärztekammer als auch die Ärztekammer Schlesw.-Holst. von der grundsätzlichen Unzulässigkeit der Abrechnung einer Besuchsgebühr neben der Leichenschaugebühr aus.

Die von der Ärztekammer Hamburg vertretene Gegenauffassung vermag nicht zu überzeugen, denn sie wird von dieser allein damit begründet, dass die derzeitige Fassung der GOÄ, welche ,,seit längerem unverändert" sei, diese Leistung nicht ,zutreffend abbilde“ das von der Ärztekammer Hamburg zur Untermauerung ihrer Rechtsansicht zitierte Urteil des AG Hannover vom 28.11.1997 - 559 C 13012/97 - enthält diesbezüglich überhaupt keine Begründung. Denn selbst wenn zutreffend sein sollte, dass die gegenwärtige Vergütung für die Leichenschau nicht auskömmlich ist - was im Ubrigen auch der Strafanzeigeerstatter so sieht - so könnte dieser Missstand doch nur durch den Gesetzgeber beseitigt werden, nicht aber durch die Ärzteschaft im Wege der Selbsthilfe.

Schließlich besteht auch ein Anfangsverdacht für ein vorsätzliches Handeln des Bf. Wie bereits dargelegt wurde, gehen sowohl die Bundesärztekammer als auch die für den Beschwerdeführer zuständige Ärztekammer Schlesw.-Holst. von der Unzulässigkeit der Abrechnung einer Besuchsgebühr für eine Leichenschau aus. Die Bundesärztekammer hat die früher von ihr vertretene Gegenauffassung revidiert und darauf in ihrem Standesorgan, dem DÄBl., im Juni 2001 ausdrücklich hingewiesen, was in einer späteren Ausgabe desselben vom November 2001 nochmals thematisiert wurde. Angesichts dessen und weil die Abrechnung einer Besuchsgebühr durch den leichenbeschauenden Arzt auch außerhalb von Fachpublikationen wiederholt als unzulässig bezeichnet wurde (vgl. dazu nur den vom 19.11.2014 stammenden Artikel mit folgender URL: http://wwvv.focus.de/panorama/welt/ leichenschau-und-totenschein-wenn-aerzte-um-dieallerletzten-euros-schachern_id_4282575.html), erscheint es durchaus naheliegend, dass der Bf. - was für eine Betrugsstrafbarkeit grundsätzlich ausreichend ist (vgl. dazu Ulsenheimer, Arztstrafrecht in der Praxis, 5. Auflage, 2015, Rdnr. 1108) - es zumindest für möglich hielt, dass ihm die geltend gemachten Honoraransprüche jedenfalls nicht in dieser Höhe zustehen.
DOI: $10.1007 / \mathrm{s} 00350-016-4518-4$

\section{Anmerkung zu LG Kiel, Beschl. v. 16.6.2016 - 10 QS 22/16 - (AG Kiel)}

\section{Markus Rothschild}

Die ärztliche Leichenschau erfüllt eine Vielzahl von relevanten Funktionen, so dass man in diesem Zusammenhang vertreten kann, dass Ärzte kraft Bestattungsgesetz mit einer hoheitlichen Aufgabe beliehen sind, und es sich bei der ärztlichen Leichenschau um ein hoheitliches Handeln im Sinne einer sonstigen Verwaltungstätigkeit handelt (Ministerium für Arbeit, Gesundheit und Soziales NW, Erlass vom 8.6.2009, Rheinisches Ärztebl. 2009, S. 57; im Gegensatz hierzu: OVG Sachsen-Anhalt, Beschl. v. 25.2.2003 - 3 L 346/00 -, juris, Rdnr. 17). Diese Wertung wird deutlich, wenn die umfangreiche Bedeutung der ärztlichen Leichenschau (vgl. Parzeller/Schmidt/Bratzke, in: Anders/Bratzke/Gotthardt/Parzeller, Die Bearbeitung von Tötungsdelikten, 2006, S. 75, 82) neben der Feststellung des Todes analysiert wird, für die Strafrechtspflege (z.B. AG Wennigsen, NJW 1989, 786, 786f.); die Gefahrenabwehr (wie zuvor und z.B. \$4 Abs. 5 BestattG Nds.); die Beweissicherung vor der Feuerbestattung; den Infektionsschutz; das Transplantationswesen.

$\mathrm{Zu}$ Sachentscheidungsvoraussetzungen für weitere Verwaltungsakte (z.B. personenstandsrechtliche Beurkundung) und die Sterbestatistik, öffentliche Gesundheitsfürsorge und der darauf beruhenden Gesundheitspolitik vgl. Ulsenheimer, in: Laufs/Kern, Handbuch des Arztrechts, 2010, \$133, Rdnr. 16; Hermanns/Filler/Roscher, GOÄ 2015, 2015, S. 135).

Die derzeitige Höhe der Vergütung der ärztlichen Leichenschau steht in einem deutlichen Widerspruch zu ihrer rechtlichen und soziomedizinischen Bedeutung (zulässiger einfacher Gebührensatz: 33,51 EUR). Sie wird als nicht mehr kostendeckend bewertet (118. Deutscher Ärztetag, 2015, Drucksache VI - 64; BÄK, Mitteilung, DÄBl. 2001, A 1711, A 1712). Bereits 2014 hat die Justizministerkonferenz gefordert: „Die Justizministerinnen und Justizminister halten auch die Erhöhung der Gebühr für die Leichenschau weiterhin für eine geeignete Maßnahme zur Verbesserung der Qualität der Leichenschau. Sie bitten daher die Gesundheitsministerkonferenz, sich im Rahmen der anstehenden Novellierung der GOÄ für eine Erhöhung dieser Gebühr einzusetzen.“ (http://justizministerium-bw.de/pb/Lde/Startseite/Ministerium/Beschluesse+de r+Herbstkonferenz+2014 Top II.5, Abrufdatum: 12.7.2016.

Prof. Dr. med. Markus Rothschild,

Institut für Rechtsmedizin, Universität zu Köln, Melatengürtel 60/62, 50823 Köln, Deutschland

\section{Unzureichende Aufklärung bei Gefahr einer Früh- geburt; Anspruch auf Schadenersatz und Schmer- zensgeld bei posttraumatischer Belastungsstörung gegenüber Krankenhausträger und Oberarzt}

GG Art. 6 Abs. 2; BGB §§249ff., 253 Abs. 2, 280, 611, 823

Abs. 1, 1626; ZPO \$286

1. Es besteht Anspruch auf Schadenersatz und Schmerzensgeld, wenn bei Gefahr einer Frühgeburt in der 23. Schwangerschaftswoche nicht hinreichend über die Behandlungschancen des Kindes aufgeklärt wurde.

Eingesandt von VRLG Dietmar Reiprich, Köln;

bearbeitet von Ass. iur. Julia Wenzel, LL.M.,

Referentin der Rechtsabteilung, Bundesärztekammer,

Postfach 1208 64, 10598 Berlin, Deutschland 